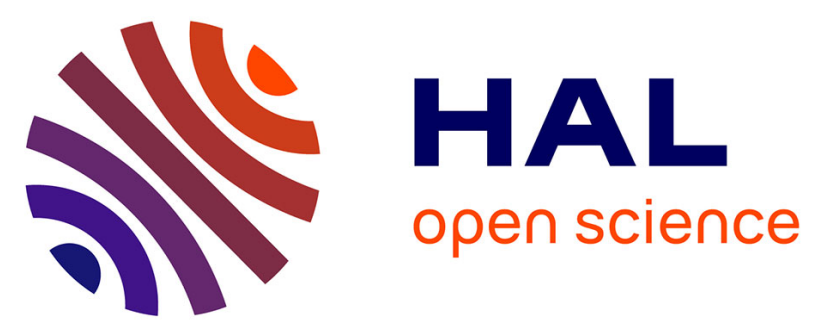

\title{
Trajectories of seasonal influenza vaccination uptake in French people with diabetes from 2006 to 2015
}

Aurélie Bocquier, Lisa Fressard, Sébastien Cortaredona, Florence Galtier, Pierre Verger

\section{- To cite this version:}

Aurélie Bocquier, Lisa Fressard, Sébastien Cortaredona, Florence Galtier, Pierre Verger. Trajectories of seasonal influenza vaccination uptake in French people with diabetes from 2006 to 2015. European Congress of Epidemiology "Crises, epidemiological transitions and the role of epidemiologists", Jul 2018, Lyon, France. pp.P8-11, 10.1016/j.respe.2018.05.401 inserm-02088962

\section{HAL Id: inserm-02088962 https://www.hal.inserm.fr/inserm-02088962}

Submitted on 3 Apr 2019

HAL is a multi-disciplinary open access archive for the deposit and dissemination of scientific research documents, whether they are published or not. The documents may come from teaching and research institutions in France or abroad, or from public or private research centers.
L'archive ouverte pluridisciplinaire HAL, est destinée au dépôt et à la diffusion de documents scientifiques de niveau recherche, publiés ou non, émanant des établissements d'enseignement et de recherche français ou étrangers, des laboratoires publics ou privés. 
P8-10

Influenza B in the general population. Retrospective analysis of French surveillance data from 2003 to 2017

A. Mosnier ${ }^{\mathrm{a}, *}$, I. Daviaud ${ }^{\mathrm{a}}$, J.-C. Soulary ${ }^{\mathrm{b}}$, S. Van der $\operatorname{Werf}^{\mathrm{c}}$, B. Lina ${ }^{\mathrm{d}}$, J.-M. Cohen ${ }^{\mathrm{a}}$

${ }^{a}$ Research department, Open Rome \& Réseau des Grog, Paris, France

${ }^{b}$ Research department, Réseau des Grog, Paris, France

${ }^{c}$ National Reference Centre for Influenza, Institut Pasteur, Paris, France

${ }^{d}$ National Reference Centre for Influenza, Hospices Civils de Lyon, Lyon, France

* Corresponding author.

E-mail address: amos@openrome.org (A. Mosnier)

Context Much attention has been paid to influenza over the past three decades with a rare focus on influenza B. Since 2000, two distinct influenza B lineages (Yamagata and Victoria) circulate alternately or simultaneously, raising the issue of potential vaccine mismatch with trivalent vaccines that contain only one of the 2 B lineages. Since 2013, WHO influenza vaccine recommendations give advice on a vaccine strain for each influenza $B$ lineage and manufacturers have developed quadrivalent seasonal vaccines containing the two lineages of influenza B viruses. In France, between 1984 and 2017, influenza surveillance in the general population has been performed by two sentinel networks, based on general practitioners and paediatricians, the Réseau national des GROG (from 1984 to 2014) and the Sentinelles network (since 2014). Practitioners of the network are providing weekly data describing their activity and collect nasal swabs in a fraction of their patients consulting for acute respiratory infection (ARI) or influenza-like infection (ILI). These swabs are sent by surface mail to the laboratories of the National Reference Centre.

Objective Our study aims to describe the circulation of the two lineages of influenza B viruses in the general population, during fourteen influenza seasons in France (2003-2017).

Methods A retrospective descriptive analysis of the GROG 2003-2013 database, completed by the Sentinelles surveillance data publicly available from 2014 was performed. Every virologically confirmed influenza positive case was included in the study, except for those with an influenza A and B co-infection. Patients whose age was unknown were also excluded.

Results and discussion Overall, 21,070 virologically confirmed influenza cases could be included, comprising 5478 influenza B cases $(26.0 \%)$. The contribution of influenza $B$ to the seasonal influenza burden varied from year-to-year. Influenza B represented more than 5\% of influenza viruses detected in eight of the fourteen studied seasons. Influenza B was considered as dominant (> 60\% of all influenza viruses of the season) in 2005-2006 (61.8\%) and 2015-2016 (71.5\%) and was co-dominant (41-60\% of all influenza viruses of the season) in 2010-2011 (47.4\%) and 2012-2013 (55.0\%). The influenza B viruses impact was mainly observed in children of the 5-14 years old group (33.0\%) but was also significant in the $\geq 65$ years old group (22.5\%). In the 8 seasons where influenza B viruses substantially circulated, both Victoria and Yamagata lineages were detected. They respectively accounted for $57.8 \%$ and $42.2 \%$ of all influenza B cases for which the lineage was available. Each lineage was predominant during four seasons: Victoria in 2005-2006, 2008-2009, 2010-2011 and 2015-2016; Yamagata in 2004-2005, 2007-2008, 2012-2013 and 2014-2015. A mismatch between the dominant circulating influenza $B$ lineage and the lineage included in the seasonal influenza vaccine was observed during four (28.5\%) of the fourteen seasons analysed, including two seasons where influenza $B$ viruses predominated (2005-2006, 2007-2008, 2008-2009 and 2015-2016). This is consistent with the results of a similar study carried out in 26 countries that reported a type B lineage mismatch in $25 \%$ of seasons. In our study, for $58.2 \%$ of the documented type B infections, the reported lineage was not included in the seasonal trivalent vaccine. Again, this is consistent with results recently published from Finland between 1999 and 2012 where $41.7 \%$ of the documented influenza B cases belonged to the mismatched lineage.

Conclusion Our results support the added value of the quadrivalent vaccine to increase vaccine effectiveness.

Disclosure of interest A. Mosnier link with: member of Sanofi influenza board, link with: member of GEIG Scientific Committee; I. Daviaud, J.-C. Soulary declare that they have no competing interest; S. Van der Werf link with: member of GEIG Scientific Committee; B. Lina link with: member of GEIG Scientific Committee, link with: financial support to attend scientific meetings by Sanofi-Pasteur and Roche; J.-M. Cohen link with: member of Astra Zeneca influenza board.

https://doi.org/10.1016/j.respe.2018.05.400

P8-11

\section{Trajectories of seasonal influenza vaccination uptake in French people with diabetes from 2006 to 2015}

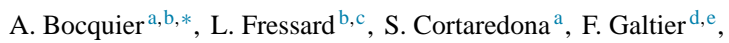
P. Verger ${ }^{\mathrm{a}, \mathrm{b}, \mathrm{e}}$

${ }^{a}$ Aix Marseille Université, IRD, AP-HM, SSA, VITROME, IHU-Méditerranée Infection, Marseille, France

${ }^{b}$ Observatoire Régional de la Santé Provence-Alpes-Côte d'Azur, Marseille, France

${ }^{c}$ Aix Marseille Université, Inserm, IRD, SESSTIM, Sciences Économiques \& Sociales de la Santé \& Traitement de l'Information Médicale, Marseille, France

${ }^{d}$ CIC 1411, CHU de Montpellier, Hôpital Saint-Éloi, Montpellier, France ${ }^{e}$ Inserm, F-CRIN, Innovative Clinical research Network in vaccinology (I-Reivac), groupe hospitalier Cochin Broca Hôtel-Dieu, Paris, France

* Corresponding author.

E-mail address: aurelie.bocquier@inserm.fr (A. Bocquier)

Introduction Seasonal influenza vaccine (SIV) is recommended in France for people aged 65 years or older and those with clinical risk factors, including diabetes. The French National Health Insurance Fund (NHIF) can identity these individuals and send them a voucher so they can obtain the vaccine free of charge. Despite this, SIV coverage remains below the target of $75 \%$ and has progressively decreased since the 2009 pandemic. We aimed to identify temporal trajectories (T) over a 10-year period of SIV uptake (SIVU) among patients with diabetes and to describe their correlates.

Methods We identified patients with diabetes in $2006(n=17,259)$ among a representative sample of French NHIF beneficiaries. We followed them from 2006 through 2015, using SIV reimbursement claims and group-based trajectory modeling to identify SIVU-T and drug reimbursement claims to assess diabetes severity and comorbidity status. A multinomial logistic regression allowed us to study characteristics associated with the SIVU-T.

Results We found 6 SIVU-T: (1) "never" $\mathrm{T}$ (prevalence: 32\%): people with quasi null SIVU probabilities ( $\leq 5 \%)$; (2) "late increasing" T (4\%): SIVU probability varying from $\leq 10 \%$ before $2011 / 12$ to $85 \%$ in $2015 / 16$; (3) "early increasing" $\mathrm{T}$ (8\%): probability varying from $\leq 10 \%$ in $2006 / 07$ to $\geq 80 \%$ starting in 2009/10; (4) "regular" $\mathrm{T}(33 \%)$ : probability always $\geq 95 \%$; (5) "progressively non-vaccinated" $\mathrm{T}(14 \%)$ : probability decreasing from $90 \%$ in 2006/07 to $20 \%$ in 2015/16; (6) "post-pandemic decreasing" T (9\%): probability decreasing right after the $2009 / 10$ season.

Overall, compared to the "never" $\mathrm{T}$ group, people in all other trajectories had poorer health at inclusion and/or over the study period (severe diabetes, high comorbidity score), people in the "late increasing" $\mathrm{T}$ group were more likely to have received newly free vaccination vouchers and/or changed general practitioners (GPs) during follow-up, and those in the "early increasing" T more likely to have received newly free vaccination vouchers and to have been hospitalized for an influenza-like illness during the follow-up. Those with "regular" $\mathrm{T}$ were slightly older than those with "never" $\mathrm{T}$ and less likely to have been hospitalized due to diabetes; those with a "progressively non-vaccinated" $\mathrm{T}$ were oldest (mean age at inclusion $=80 \pm 7$ years versus $65 \pm 14$ in the study population). Finally, people with a "post-pandemic decreasing" $T$ were more frequently women, more likely to have been hospitalized due to an influenza-like illness, and to have changed GPs during the follow-up.

Conclusions SIVU behavior was stable in most people with diabetes over the study period: one third were vaccinated regularly and another third never. The latter were globally healthier than the other groups and may feel less vulnerable to it, despite their clinical risk. About $25 \%$ became less inclined toward vaccination in one of two different patterns: the "progressively non-vaccinated" $\mathrm{T}$ may reflect the patient and/or healthcare professional's doubts about the benefits of SIV after a certain age (in part due to immunoscenescence); those in 
the "post-pandemic decreasing" $\mathrm{T}$ may have lost confidence in SIV after controversies in France during the 2009 mass vaccination campaign against the pandemic. About $10 \%$ became more likely to be vaccinated during the study period; our results suggest that receiving free vouchers for the first time might have triggered or fostered this behavioral change. Changing GPs and hospitalization for an influenza-like illness were associated with both increasing and decreasing trajectories. These events may represent key opportunities to foster or prevent behavioral changes toward SIV. Further research is needed to better understand the chronology of these events and potential causal pathways. These results should help stakeholders to adapt public health interventions to specific subgroups.

Disclosure of interest The authors declare that they have no competing interest.

https://doi.org/10.1016/j.respe.2018.05.401

\section{P8-13}

\section{Demand for family planning satisfied among adolescents by marital status and parity: An analysis of $73 \mathrm{low}$ and middle-income countries}

C. Coll*, A. Barros, F. Ewerling, F. Hellwig

International Center for Equity in Health, Federal University of Pelotas, Pelotas, Brazil

* Corresponding author.

E-mail address: ccoll@equidade.org (C. Coll)

Introduction Despite the worldwide positive trends in contraceptive use and family planning indicators over the last decades, progress among adolescent women has been happening in a slower pace, and the demand for family planning satisfied remains very low in this populational group. Social-cultural and structural barriers often prevent adolescents from achieving their reproductive desires, which can result in unintended and unhealthy pregnancies. The aim of the present study is to describe the demand for family planning satisfied with modern methods (mDFPS) among adolescents aged $15-19$ by marital status and parity in low and middle-income countries (LMICs).

Methods We obtained data from nationally representative surveys (Demographic and Health Surveys and Multiple Indicator Surveys) with public available datasets carried out since 2005 . We use the most recently collected data from any country. The present analyses are based on currently sexually active adolescent women aged 15-19 years. mDFPS was defined as the proportion of women in need of contraception that are currently using a modern contraceptive method (IUD, implants, pill, injectable, diaphragm, condom, foam or jelly, patch, emergency contraception; and male and female sterilization). Women in need of contraception are those who are fecund and do not want to become pregnant within the next two years or are unsure if or when they want to become pregnant. Pregnant women with a mistimed or unwanted pregnancy are also considered in need of contraception. We estimated the mean mDFPS coverage for each country and world region by adolescent group defined by marital status and parity (married $1+$ children, married no children, not married sexually active). Analyses are presented by world region following the UNICEF classification. All analyses took into account the multistage sampling strategies and sample weights.

Results A total of 73 LMICs with available information for sexually active adolescents were included in this analysis ( 9 from the CEE and the CIS, 8 from the East Asia and the Pacific, 16 from the Eastern and Southern Africa, 16 from the Latin America and Caribbean, 3 from South Asia and, 21 from the West and Central Africa). Adolescents who were married with no children presented the lowest mean mDFPS coverage in all world regions when compared to married adolescents with one or more children and those who were not married. mDFPS coverage ranged from $12.8 \%$ in West and Central Africa to $41.6 \%$ in Latin America and Caribbean among not married with no children adolescents; from $18.8 \%$ in West and Central Africa to $60.5 \%$ in Latin America and Caribbean among married with one or more children adolescents and, from $19.4 \%$ in East Asia and the Pacific to $73.9 \%$ in CEE and the CIS among not married sexually active adolescents. mDFPS among married adolescents with no children was below $20 \%$ in 32 of the 73 the low and middle-income countries analysed, of which 14 presented a mDFPS below $10 \%$ (8 of them belonging to the West and Central Africa).

Conclusion Overall, we found that most of the girls who wanted to delay, or limit pregnancy were not using a modern contraceptive method. In all world regions, the lowest mDFPS coverage was found for married adolescents with no children. In this sense, priority countries for interventions are those belonging to the West and Central Africa region. Global efforts to prevent unintended pregnancies and improve pregnancy spacing among adolescents should consider the existing social norms regarding marriage and fertility expectations so that family planning strategies can effectively reach adolescents in these countries.

Disclosure of interest The authors declare that they have no competing interest.

https://doi.org/10.1016/j.respe.2018.05.403

\section{P8-14}

The global influenza hospital surveillance network (GIHSN), a worldwide platform for timely generation of severe influenza epidemiological data

C. El Guerche-Séblain ${ }^{\mathrm{a}}$, C. Commaille-Chapus ${ }^{\mathrm{b}, *}$, M. Morizet $^{\mathrm{b}}$, J.-Y. Robin ${ }^{\mathrm{b}}$, C. Mahé ${ }^{\mathrm{a}}$

${ }^{a}$ Global Vaccines Epidemiology and Modeling, Sanofi Pasteur, Lyon, France

${ }^{b}$ Strategy, Open Health Company, Paris, France

* Corresponding author.

E-mail address: clotilde.elguercheseblain@ sanofi.com (C.Commaille-Chapus)

Introduction According to World Health Organization (WHO) estimates, annual influenza epidemics are estimated to result in about 3 to 5 million cases of severe illness, and about 290,000 to 650,000 deaths worldwide. While policy makers are expected to place higher value on vaccines indicated for prevention of severe illness, high quality global data on severe influenza are scarce. This is further complicated by the variability of the viruses and the severity of influenza epidemics between years and geographical areas. The Global Influenza Hospital Surveillance Network (GIHSN) supported by the Foundation for Influenza Epidemiology is a platform to generate such important public health data.

Methods The GIHSN consists of a network of country sites affiliated with public health authorities coordinating several hospitals. This multicenter, prospective, hospital-based active surveillance, is coordinated by the Open Health Company and funded by the Foundation for Influenza Epidemiology created by Sanofi Pasteur. A standard protocol is shared between sites allowing for comparison and pooling of data across sites. Patients hospitalized during the influenza season are asked for recent (less than 7 days old) influenza-likeillness (ILI) symptoms before admission. All consenting ILI cases are swabbed and tested by multiplex real-time polymerase chain reaction (RT-PCR) for influenza. Influenza positive RT-PCR samples are sub-typed to identify A/H1N1, $\mathrm{A} / \mathrm{H} 3 \mathrm{~N} 2$ strain subtypes or B/Yamagata, B/Victoria lineages. When vaccine uptake allows, vaccine effectiveness is estimated using a test negative design method. Sites are invited to share their data through an online collection tool. Data are then aggregated, and indicators are displayed using state-of-the-art data visualization techniques on the network website www.gihsn.org. Data are managed through an associative engine, which can combine a very large number of data sources and indexes every possible relationship in the data. Users are not restricted to linear exploration within partial views of data and can gain immediate insights and explore data in multiple directions.

Results The GIHSN has been progressively scaled up and has generated data for six consecutive seasons, for both Northern and Southern hemisphere, representing now a yearly sample of more than 12,000 individual samples tested by RT-PCR with detailed demographic, clinical and virological data. During the 2016-2017 season, close to 3000 cases of hospitalizations from influenza have been documented. Type of data generated include influenza activity and lengths of epidemics, pattern of strain circulation by subtype by region, burden of severe laboratory confirmed influenza for various populations, analyses of disease risk factors and vaccine effectiveness estimates. Genetic strain sequencing characterization is also generated locally. Results are published yearly in peer reviewed scientific journals and presented in international conferences. For the 2017-2018 season, the GIHSN expanded to more than 40 hospitals in 20 countries. 